Short communication

\title{
Switching on the deep brain stimulation: Effects on cardiovascular regulation and respiration ${ }^{\text {is }}$
}

\author{
S. Vigneri ${ }^{\text {a,* }}$, P. Guaraldi ${ }^{\text {b }}$, G. Calandra-Buonaura ${ }^{\text {b }}$, R. Terlizzi ${ }^{\text {b }}$, A. Cecere ${ }^{\text {b }}$, G. Barletta ${ }^{\text {b }}$, P. Cortelli ${ }^{\text {b }}$ \\ a Department of Clinical Neurosciences, University of Palermo, Palermo, Italy \\ ${ }^{b}$ Department of Neurological Sciences, University of Bologna, Bologna, Italy
}

\section{A R T I C L E I N F O}

Article history:

Received 11 May 2011

Received in revised form 29 July 2011

Accepted 13 September 2011

\section{Keywords:}

Cardiovascular

Deep brain stimulation

Blood pressure

Heart rate

Respiratory

Parkinson

Cluster

\begin{abstract}
A B S T R A C T
Background: Objective of this study was to evaluate the acute cardiovascular and respiratory effects of switching on the deep brain stimulation in the follow up of nine Parkinson's disease patients with subthalamic nucleus stimulation and six cluster headache patients with posterior hypothalamic area stimulation. Methods: Systolic and diastolic blood pressure, heart rate, and respiratory rate were monitored continuously during supine rest in both groups. Each patient was assessed in two conditions: resting supine with stimulator off and with stimulator on.

Results: In supine resting condition switching on the DBS induced no significant changes $(p>0.05)$ in systolic and diastolic blood pressure as well as in heart rate and respiratory rate, in both groups of patients, either taking 1 min or 10 heartbeats as a sample for analysis.

Conclusions: Switching on the DBS does not modify heart rate, blood pressure nor respiratory rate in both Parkinson and cluster headache patients under resting conditions.
\end{abstract}

(C) 2011 Elsevier B.V. All rights reserved.

\section{Introduction}

The subthalamic nucleus (STN) is a well known target area for DBS in PD, especially in patients with advanced stages poorly responsive to drug therapy (Kumar et al., 1998). The intra-operative stimulation of STN, and the acute effect of switching on the stimulator 6 months after the implantation, have been shown to produce, not only motorrelated responses, but also autonomic responses both in animals and humans (mainly a conspicuous increase of heart rate) (Angyan and Angyan, 1999; Sauleau et al., 2005).

The posterior hypothalamic area (PHA) stereotactic DBS was proved to be a successful treatment for chronic drug-resistant cluster headache $(\mathrm{CH})$ patients (Sano et al., 1970). The PHA is involved in the control of sleep-wake cycle (Lin et al., 1989), cardiovascular regulation (Martin et al., 1991) and the expression of defensive-aggressive behavior (Shekhar and DiMicco, 1987). Early experimental animal studies demonstrated that electrical or chemical stimulation of posterior hypothalamus increases respiration frequency, heart rate, arterial pressure and elicits a redistribution of organ blood flow similar to that occurring during voluntary exercise (Hess, 1969). More recent studies pointed at the

\footnotetext{
is Financial disclosures: none of the authors have any financial interest to disclose.

* Corresponding author at: Department of Clinical Neurosciences, University of Palermo, Via Gaetano La Loggia 1, 90129, Palermo (PA), Italy. Tel.: + 39091 6555101; fax: + 390916555102 .

E-mail address: simone.vigneri@gmail.com (S. Vigneri).
}

posterior hypothalamus as an area that integrates information from contracting muscles with central command to generate the necessary responses to exercise (Waldrop and Stremel, 1989; Dampney et al., 2002). Few stimulation studies in humans are available. A previous study reported a rise in blood pressure, tachycardia and pupillary dilation while performing a therapeutic posterior hypothalamus stimulation in pathologically aggressive patients (Sano et al., 1970). The new application of PHA DBS for chronic drug-resistant $\mathrm{CH}$ patients has provided a new unique opportunity to study the role of this structure on cardiovascular autonomic regulation in humans (May et al., 1999). Another scientific work described polypnoea, tachycardia and moderate hypertension during the implantation procedure of one cluster headache patient with concomitant panic sensation (Schoenen et al., 2005). Although, no autonomic effects were reported by the Milano's group in patients undergoing intra-operative stimulation of posterior hypothalamic area up to $4 \mathrm{~V}$ (Franzini et al., 2003).

Stimulated by these discrepancies, although observed in different settings, having the opportunity to test both patients with $\mathrm{PD}$ and $\mathrm{CH}$ treated with DBS of STN and PHA respectively during their postoperative follow-up, we evaluated the acute effects on their cardiovascular system by switching on the stimulator.

\section{Patients}

Nine patients with PD (six males, mean age: $58.7 \pm 9$ ) with bilateral DBS of STN and six CH patients (five males, mean age: $36.2 \pm 9.9$ ) with 
monolateral DBS of PHA were enrolled in the study. The difference between the mean ages of the two groups was significant $(p<0.05)$.

The patients' inclusion/exclusion criteria and the surgical technique were previously described (Broggi et al., 2003; Franzini et al., 2003; Leone et al., 2004; Machado et al., 2006). The accuracy of electrode placement was checked by means of a post stereotactic procedure MRI, while the voltage pulse width and the frequency of the stimulator were optimized for the best clinical results by the scientists from Gemelli Hospital (Rome, Italy) and Besta Institute (Milan, Italy) [Table 1].

\section{Protocol}

$\mathrm{PD}$ and $\mathrm{CH}$ patients were assessed in our Autonomic Unit $22 \pm$ 22 months (range 1-35) and $2 \pm 1$ months (range 0.8-3.9) after implantation respectively.

All patients were evaluated in a temperature-controlled $\left(23 \pm 1{ }^{\circ} \mathrm{C}\right)$ clinical investigation room between 8 and 12 o'clock in the morning in two different conditions: 1) resting supine with stimulator off and 2) resting supine with stimulator on.

Before the assessment patients were allowed to drink water but otherwise fasted overnight. All had to abstain from smoking or drinking alcohol on the day before the study. For ethical reason patients were free to assume their usual medications with the exception of the morning they performed the autonomic tests. Patients were asked not to sleep or talk during the study.

Systolic and diastolic blood pressure (SBP, DBP; Portapres model 2, TNO-TPD Biomedical Instrumentation, Delft, the Netherlands), heart rate (HR; Grass 7P511 [Astro-Med West Warwick, RI, USA] and Light Work Station for digital R-R quantification), oronasal and abdominal breathing (Grass DC preamplifier 7P1) were monitored continuously. Data were initially acquired for 2 hours in off condition, then the stimulator was turned on and new acquisitions were obtained. All the participants were aware about the time when the stimulator was switched or whether it was switched on or off.

All patients gave their informed consent prior to their inclusion in the study and the research protocol was approved by the Institutional Review Board committees from the University of Bologna.

Data from the last minute in stimulator off were compared to the first minute with stimulator on and data from the last 10 heartbeats with stimulation off were compared to the 10 heartbeats with stimulator on by repeated-measures $t$-tests. The acute effects of switching on the DBS in PD versus $\mathrm{CH}$ patients were compared using the Bonferroni/Dunn test. A $p$ value $<0.05$ was considered significant.

\section{Results}

In supine resting condition switching on the DBS induced no changes in systolic (SBP), diastolic (DBP), heart rate (HR: calculated as mean $\mathrm{R}-\mathrm{R}$ intervals), and respiratory rate (RR).

After switching on the stimulator, no significant changes in SBP were observed in the $\mathrm{PD}$ and $\mathrm{CH}$ patients, neither considering the first minute or the first 10 heartbeats to the previous minute or 10 heartbeats respectively $(p>0.05)$ [Table 2].

No significant differences were observed in DBP after switching on the DBS in the two groups, neither considering the first minute or the first 10 heartbeats as a sample for analysis $(p>0.05)$ [Table 2].

Mean R-R intervals (RRI) during the first 10 beats or the first minute with stimulator on were not significantly different if compared to the previous 10 heartbeats or minute during stimulator off respectively, neither in the PD nor $\mathrm{CH}$ patients $(p>0.05)$ [Table 2].

Finally within the first minute of stimulation there were no significative changes in RR in PD and CH patients $(p>0.05)$ [Table 2].

\section{Discussion}

This study shows that in supine resting condition acute switching on the DBS in the follow up of Parkinson's and cluster headache patients does not affect significantly systolic and diastolic blood pressure, heart and respiratory rate in both groups either considering the first minute or the 10 heartbeats following the stimulation.

A previous study demonstrated a significant HR increase during the first 10 heartbeats after switching on the DBS in three PD patients, whereas BP and RR did not show any significant variation (Kaufmann et al., 2002). Nevertheless in this study patients were unaware of either the time when the stimulator was switched or whether it was switched on or off, and this discrepancy could represent the effect of an unspecific arousal, and not being due to the acute effect of DBS stimulation "per se." In our experience, even if the patients are blind to the DBS status they can "feel" the exact moment in which the stimulator is switched on. Furthermore the small sample of patients and the lack of an adequate baseline monitoring represent important limitations of the above-mentioned study.

Furthermore a more recent work has shown that PD patients both in basal conditions and during DBS present different autonomic pattern of response according to the site of stimulation: patients who underwent stimulation of the dorsalmost region, produced changes in $\mathrm{R}-\mathrm{R}$ intervals that were constant over time regardless of the patients' awareness. By contrast, the stimulation of the ventral region produced autonomic and emotional responses that were inconstant

Table 1

Details of PD and $\mathrm{CH}$ patients and their corresponding stimulator models, localization and set parameters.

\begin{tabular}{|c|c|c|c|c|c|c|c|c|c|c|c|c|c|c|}
\hline \multirow[t]{2}{*}{ Patients } & \multirow{2}{*}{$\frac{\text { Age }}{\text { Years }}$} & \multirow[t]{2}{*}{ Sex } & \multirow[t]{2}{*}{ Stimulation } & \multirow[t]{2}{*}{ Model } & \multicolumn{5}{|c|}{ Left side } & \multicolumn{5}{|c|}{ Right side } \\
\hline & & & & & $\begin{array}{l}\text { Amp } \\
(\mathrm{V})\end{array}$ & $\begin{array}{l}\text { Wide } \\
(\mathrm{Ms})\end{array}$ & $\begin{array}{l}\text { Freq } \\
(\mathrm{Hz})\end{array}$ & Polarity & Mode & $\begin{array}{l}\text { Amp } \\
(\mathrm{V})\end{array}$ & $\begin{array}{l}\text { Wide } \\
(\mathrm{Ms})\end{array}$ & $\begin{array}{l}\text { Freq } \\
(\mathrm{Hz})\end{array}$ & Polarity & Mode \\
\hline PD 1 & 52 & M & Bilateral & Kinetra & 2.40 & 60 & 185 & $\mathrm{el}-0$ & Cont. & 2.20 & 60 & 185 & el -7 & Cont. \\
\hline PD 2 & 56 & M & Bilateral & Kinetra & 2.20 & 60 & 185 & $\mathrm{el}-3$ & Cont. & 2.20 & 60 & 185 & el -6 & Cont. \\
\hline PD 3 & 44 & $\mathrm{~F}$ & Bilateral & Kinetra & 2.85 & 60 & 195 & el -1 & Cont. & 2.65 & 60 & 195 & $\mathrm{el}-5$ & Cont. \\
\hline PD 4 & 67 & M & Bilateral & Kinetra & UNK & UNK & UNK & UNK & UNK & UNK & UNK & UNK & UNK & UNK \\
\hline PD 5 & 53 & $\mathrm{~F}$ & Bilateral & Kinetra & UNK & UNK & UNK & UNK & UNK & UNK & UNK & UNK & UNK & UNK \\
\hline PD 6 & 54 & M & Bilateral & Kinetra & 2.30 & 60 & 185 & el -0 & Cont. & 2.60 & 60 & 185 & el $-4-5$ & Cont. \\
\hline PD 7 & 70 & M & Bilateral & Kinetra & 3.20 & 90 & 180 & el -2 & Cont. & 3.00 & 90 & 180 & el -6 & Cont. \\
\hline PD 8 & 66 & $\mathrm{~F}$ & Bilateral & Kinetra & 2.70 & 60 & 160 & $\mathrm{el}-0-1$ & Cont. & 2.70 & 60 & 160 & el $-4-5$ & Cont. \\
\hline PD 9 & 67 & M & Bilateral & Kinetra & 2.90 & 60 & 140 & $\mathrm{el}-1$ & Cont. & 2.95 & 60 & 140 & $\mathrm{el}-5$ & Cont. \\
\hline $\mathrm{CH} 1$ & 30 & M & Unilateral & Soletra 7426 & 1.3 & 60 & 185 & UNK & Cont. & NA & NA & NA & NA & NA \\
\hline $\mathrm{CH} 2$ & 45 & M & Unilateral & Soletra 7427 & 2.8 & 90 & 130 & UNK & Cont. & NA & NA & NA & NA & NA \\
\hline $\mathrm{CH} 3$ & 27 & $\mathrm{~F}$ & Unilateral & Soletra 7428 & 1.6 & 90 & 180 & $C(+)-3(-)$ & Cont. & NA & NA & NA & NA & NA \\
\hline $\mathrm{CH} 4$ & 25 & M & Unilateral & Soletra NFW625261 & NA & NA & NA & NA & NA & 2.1 & 60 & 185 & $C(+)-1(-)$ & Cont. \\
\hline $\mathrm{CH} 5$ & 43 & M & Unilateral & Kinetra NFD624515 & 2.6 & 60 & 180 & UNK & Cont. & NA & NA & NA & NA & NA \\
\hline $\mathrm{CH} 6$ & 47 & M & Unilateral & Kinetra NFD624570 & NA & NA & NA & NA & NA & 1.8 & 60 & 180 & $C(+)-1(-)$ & Cont. \\
\hline
\end{tabular}

Amp: amplitude. $\mathrm{CH}$ : cluster headache. Cont.: continuous. el: electrode. Freq: frequency. NA: not applicable. PD: Parkinson disease. UNK: unknown data. 
Table 2

Switching the stimulator on does not affect cardiovascular nor respiratory parameters in patients with Parkinson's disease and cluster headache.

\begin{tabular}{|c|c|c|c|c|}
\hline & & & PD patients & $\mathrm{CH}$ patients \\
\hline \multirow[t]{6}{*}{ RRI } & $1 \mathrm{~min}$ & OFF (s) & $0.868 \pm 0.15$ & $0.877 \pm 0.07$ \\
\hline & & ON (s) & $0.863 \pm 0.15$ & $0.846 \pm 0.05$ \\
\hline & & OFF $v s$. ON $p$-value & NS & NS \\
\hline & 10 beats & $\mathrm{OFF}(\mathrm{s})$ & $0.868 \pm 0.15$ & $0.883 \pm 0.07$ \\
\hline & & ON (s) & $0.870 \pm 0.15$ & $0.889 \pm 0.08$ \\
\hline & & OFF vs. ON $p$-value & NS & NS \\
\hline \multirow[t]{6}{*}{ SBP } & $1 \mathrm{~min}$ & OFF (mmHg) & $135 \pm 14$ & $119 \pm 12$ \\
\hline & & $\mathrm{ON}(\mathrm{mmHg})$ & $135 \pm 17$ & $119 \pm 11$ \\
\hline & & OFF $v s$. ON $p$-value & NS & NS \\
\hline & 10 beats & OFF (mmHg) & $135 \pm 14$ & $119 \pm 12$ \\
\hline & & $\mathrm{ON}(\mathrm{mmHg})$ & $135 \pm 14$ & $121 \pm 11$ \\
\hline & & OFF vs. ON $p$-value & NS & NS \\
\hline \multirow[t]{6}{*}{ DBP } & $1 \mathrm{~min}$ & OFF (mmHg) & $73 \pm 8$ & $65 \pm 10$ \\
\hline & & $\mathrm{ON}(\mathrm{mmHg})$ & $73 \pm 9$ & $65 \pm 10$ \\
\hline & & OFF $v s$. ON $p$-value & NS & NS \\
\hline & 10 beats & $\mathrm{OFF}(\mathrm{mmHg})$ & $73 \pm 8$ & $65 \pm 10$ \\
\hline & & ON (mmHg) & $73 \pm 8$ & $65 \pm 9$ \\
\hline & & OFF vs. ON $p$-value & NS & NS \\
\hline \multirow[t]{6}{*}{$\mathrm{RR}$} & $1 \mathrm{~min}$ & OFF (breaths/m) & $24 \pm 6$ & $18 \pm 4$ \\
\hline & & ON (breaths/m) & $23 \pm 6$ & $17 \pm 4$ \\
\hline & & OFF vs. ON $p$-value & NS & NS \\
\hline & 10 beats & OFF (breaths/m) & NA & NA \\
\hline & & ON (breaths/m) & NA & NA \\
\hline & & OFF vs. ON $p$-value & NA & NA \\
\hline
\end{tabular}

DBP: diastolic blood pressure. mmHG: millimeters of mercury. NA: not applicable. NS: not significant. SBP: systolic blood pressure. RR: respiratory rate. RRI: r-r interval. s: seconds.

over time and different responses were elicited with hidden and open stimulations (Benedetti et al., 2004).

Possible confounders compared to prior studies must be taken in account; the intensity of stimulation may surely influence the autonomic responses by spreading the stimulus to nearby structures but even if in our Parkinson's patients the mean intensity $(2.6 \pm 0.3 \mathrm{~V})$ was slightly higher than in Kauffmann's work $(2.3 \pm 0.5 \mathrm{~V})$ we did not observe any change in heart rate; therefore it is unlikely that intensity influenced our results.

The finding of cardiovascular and autonomic responses following STN and the surrounding areas stimulation have been described both in animals (Angyan and Angyan, 1999) and humans (Priori et al., 2001); it seems to involve complex relationships between central and peripheral areas. The basal ganglia project to several nuclei that may modify autonomic outflow. High frequency stimulation has been proven to inhibit neuronal activity around the implanted electrodes and this effect on fibers and cell bodies does not allow to ascertain the neurons involved in the pattern (Beurrier et al., 2001) and, consequentially, the exact role of nearby structures on autonomic outflow.

With regard to the timing of post-implantation evaluation, a wider time interval between electrodes positioning and testing could be associated with a more serious neuronal degeneration and, consequentially, a blunted autonomic response. This could play a role in a long-term perspective, although, a previous work (Erola et al., 2006) evaluated Parkinson's patients after a shorter period than we did (i.e. 1 year) showing no autonomic function changes post-DBS; therefore it is unlikely that this factor may have affected our results.

Studies evaluating the effects of acute switching on of the deep brain stimulator in the follow-up of $\mathrm{CH}$ patients are missing. Previous intraoperative studies are limited and led to conflicting results, probably because of differences in both the targeting and the stimulation parameters (Franzini et al., 2003; Schoenen et al., 2005).

This study demonstrates for the first time that in supine resting condition, acute switching on of the DBS in the follow up of PD and $\mathrm{CH}$ patients does not affect significantly SBP, DBP, HR and RR in PD and $\mathrm{CH}$ patients, despite their different pathologies and stimulation site and technique, suggesting that the complex mechanisms that maintain autonomic parameters around the "set point" in basal condition are not affected in our patients.

Some unavoidable limitations of our study should be addressed: first we could not obtain information about ventral or dorsal placement of PD patients stimulator which could give rise to different autonomic responses after switching on the DBS; second, we could not ask the patients to withdraw their usual medication for ethical considerations, and this could have influenced the autonomic response; last, we cannot exclude a main direct primary disease or medication effect, affecting the results. Nevertheless, although theoretically possible, we did not observe any abnormality in autonomic control of cardiovascular reflexes in both groups of patients.

\section{Conclusions}

No significant change in heart rate, blood pressure nor respiratory rate was appreciated in both Parkinson and cluster headache patients after switching on the stimulator either considering $1 \mathrm{~min}$ or 10 heartbeats as a sample for analysis. A possible explanation could be that our patients were aware of either the time when the DBS was switched or whether it was turned on or off. Noteworthy, our patients were tested in supine resting conditions almost 2 years post implantation; this may explain the differences between our results and previous studies.

\section{Conflict of interest statement}

The authors have no financial interest to disclose.

\section{Acknowledgments}

We thank Dr. A. Albanese, Dr. G. Broggi, Prof. G. Bussone, Dr. A. Franzini and Dr. M Leone for patient referral.

\section{References}

Angyan, L., Angyan, Z., 1999. Subthalamic influences on the cardiorespiratory functions in the cat. Brain Res. 847 (1), 130-133.

Benedetti, F., Colloca, L., Lanotte, M., Bergamasco, B., Torre, E., Lopiano, L., 2004. Autonomic and emotional responses to open and hidden stimulations of the human subthalamic region. Brain Res. Bull. 63 (3), 203-211.

Beurrier, C., Bioulac, B., Audin, J., Hammond, C., 2001. High-frequency stimulation produces a transient blockade of voltage-gated currents in subthalamic neurons. J. Neurophysiol. 85, 1351-1356.

Broggi, G., Franzini, A., Marras, C., Romito, L., Albanese, A., 2003. Surgery of Parkinson's disease: inclusion criteria and follow-up. Neurol. Sci. 24 (Suppl. 1), S38-S40.

Dampney, R.A., Coleman, M.J., Fontes, M.A., et al., 2002. Central mechanisms underlying short- and long-term regulation of the cardiovascular system. Clin. Exp. Pharmacol. Physiol. 29 (4), 261-268.

Erola, T., Haapaniemi, T., Heikkinen, E., et al., 2006. Subthalamic nucleus deep brain stimulation does not alter long-term heart rate variability in Parkinson's disease. Clin. Auton. Res. 16 (4), 286-288 Aug.

Franzini, A., Ferroli, P., Leone, M., Broggi, G., 2003. Stimulation of the posterior hypothalamus for treatment of chronic intractable cluster headaches: first reported series. Neurosurgery 52 (5), 1095-1099 discussion 1099-1101.

Hess WR. Hypothalamus and thalamus. Experimental documentation. 2nd enl. ed. Stuttgart,: Thieme, 1969.

Kaufmann, H., Bhattacharya, K.F., Voustianiouk, A., Gracies, J.M., 2002. Stimulation of the subthalamic nucleus increases heart rate in patients with Parkinson disease. Neurology 59 (10), 1657-1658.

Kumar, R., Lozano, A.M., Kim, Y.J., et al., 1998. Double-blind evaluation of subthalamic nucleus deep brain stimulation in advanced Parkinson's disease. Neurology 51 (3), 850-855.

Leone, M., May, A., Franzini, A., et al., 2004. Deep brain stimulation for intractable chronic cluster headache: proposals for patient selection. Cephalalgia 24 (11), 934-937.

Lin, J.S., Sakai, K., Vanni-Mercier, G., Jouvet, M., 1989. A critical role of the posterior hypothalamus in the mechanisms of wakefulness determined by microinjection of muscimol in freely moving cats. Brain Res. 479 (2), 225-240.

Machado, A., Rezai, A.R., Kopell, B.H., Gross, R.E., Sharan, A.D., Benabid, A.L., 2006. Deep brain stimulation for Parkinson's disease: surgical technique and perioperative management. Mov. Disord. 21 (Suppl. 14), S247-S258. 
Martin, J.R., Knuepfer, M.M., Westfall, T.C., 1991. Hemodynamic effects of posterior hypothalamic injection of neuropeptide Y in awake rats. Am. J. Physiol. 261 (3 Pt 2), H814-H824.

May, A., Ashburner, J., Buchel, C., et al., 1999. Correlation between structural and functional changes in brain in an idiopathic headache syndrome. Nat. Med. 5 (7), 836-838.

Priori, A., Cinnante, C., Genitrini, S., et al., 2001. Non-motor effects of deep brain stimulation of the subthalamic nucleus in Parkinson's disease: preliminary physiological results. Neurol. Sci. 22, 85-86.

Sano, K., Mayanagi, Y., Sekino, H., Ogashiwa, M., Ishijima, B., 1970. Results of stimulation and destruction of the posterior hypothalamus in man. J. Neurosurg. 33 (6), 689-707.
Sauleau, P., Raoul, S., Lallement, F., et al., 2005. Motor and non motor effects during intraoperative subthalamic stimulation for Parkinson's disease. J. Neurol. 252 (4), 457-464.

Schoenen, J., Di Clemente, L., Vandenheede, M., et al., 2005. Hypothalamic stimulation in chronic cluster headache: a pilot study of efficacy and mode of action. Brain 128 (Pt 4), 940-947.

Shekhar, A., DiMicco, J.A., 1987. Defense reaction elicited by injection of GABA antagonists and synthesis inhibitors into the posterior hypothalamus in rats. Neuropharmacology 26 (5), 407-417.

Waldrop, T.G., Stremel, R.W., 1989. Muscular contraction stimulates posterior hypothalamic neurons. Am. J. Physiol. 256 (2 Pt 2), R348-R356. 